Rossi, A, de Cataldo, S., Di Michele, V., et al (1990) Neurological soft signs in schizophrenia. British Journal of Psychiatry, 157, 735-739.

Department of Psychiatry Institute of Experimental Medicine University of L'Aquila 67100 L'Aquila, Italy

\section{Lethal catatonia and NMS}

SIR: We would like to make some remarks in connection with the two letters by Tan \& Ong (Journal, June 1991, 158, 858 and November 1991, 159, 729-730).

They suggested that the mother and the younger daughter in our paper on the familial occurrence of neuroleptic malignant syndrome (NMS) (Journal, June 1991, 158, 850-853) could be diagnosed as lethal catatonia (probably they meant that of psychogenic origin) rather than NMS, since they had past histories of catatonia. However, we believe that the episodes presented were neuroleptic-induced, e.g. the first episode of the mother, which was not preceded by catatonia, developed within eight days of the initiation of neuroleptic treatment.

More importantly, Tan \& Ong appear to consider NMS and lethal catatonia (LC) as separate entities, despite the following discussions suggesting that NMS is a subtype of LC; Gelenberg (1976), and Barnes et al (1986) emphasised that catatonia was a syndrome with various causes, and neuroleptics could cause catatonic state. Mann et al (1986) emphasised that LC was also a syndrome rather than a specific disease based on a comprehensive review, and suggested that NMS was a neuroleptic-induced iatrogenic form of LC. More recently, White \& Robins (Journal, March 1991, 158, 419-421) described five cases of NMS in which catatonia preceded the syndrome. This paper and our paper indicate that a patient with a past history of catatonia is at high risk of developing NMS, suggesting that NMS and LC have a common pathogenesis, probably hypodopaminergic function.

Tan \& Ong suggested that NMS and LC should be differentiated, since the treatment of LC would be the continuation of neuroleptics and ECT. However, as reviewed by Mann et al (1986), neuroleptics are generally inadequate in treating LC and, in fact, may aggravate or complicate the disorder. The two case reports by Kish et al (1990) illustrate this view; these two cases, in which clinical pictures were indistinguishable from NMS, were diagnosed as LC, and neuroleptics were continued, which ended in death despite electroconvulsive therapy (ECT). This finding suggests that neuroleptics should be discontinued in the conditions now labelled as NMS or LC, regardless of which diagnosis is given. Incidentally, Tan \& Ong misquoted Mann et al (1986) who suggested the discontinuation of neuroleptics whenever LC was suspected, for the reasons mentioned above. The report by Mahmood (Journal, March $1991,158,437-438$ ) on the effectiveness of bromocriptine for catatonic stupor, and the review by Davis et al (1991) on the effectiveness of ECT for NMS also suggest that the two disorders respond to common measures.

Our paper on the familial occurrence of NMS suggests that the predisposition to this syndrome may be genetically transmitted. This further suggests the close linkage between NMS and LC, since the familial tendency to catatonia (including LC) has also been reported (Barnes et al, 1986).

Thus, it is far more practical to consider NMS as a subtype of LC than to consider them as separate entities for the understanding and management of these disorders.

Barnes, M. P., Saunders, M., Walls, T. J., el al (1986) The syndrome of Karl Ludwig Kahlbaum. Journal of Neurology, Neurosurgery, and Psychiatry, 49, 991-996.

Davis, J. M., Janicak, P. G., Sakkas, P., el al (1991) Electroconvulsive therapy in the treatment of the neuroleptic malignant syndrome. Convulsive Therapy, 7, $111-120$.

GeLENBERG, A. J. (1976) The catatonic syndrome. Lancet, $i$, 1339-1341.

Kish, S. J., Kleinert, R., Minauf, M., et al (1990) Brain neurotransmitter changes in three patients who had a fatal hyperthermia syndrome. American Journal of Psychiatry, 147, 1358-1363.

ManN, S. C., Caroff, S. N., Bleier, H. R., el al (1986) Lethal catatonia. American Journal of Psychiatry, 143, 1374-1381.

KOICHI OTANI
SUNAO KANEKO
YUTAKA FUKUSHIMA
$\begin{aligned} & \text { Department of Neuropsychiatry } \\ & \text { Hirosaki University Hospital } \\ & \text { Hirosaki 036, Japan }\end{aligned}$

\section{Pisa syndrome-a confusing term}

SIR: I read with interest the article by Turk \& Lask ("Pisa syndrome in an adolescent on neuroleptic medication", Journal, March 1991, 158, 422-423). The case report, concerning a 15-year-old girl, and the discussion are important because they describe an impressive acute dystonic reaction which could have been mistaken for malingering or naughtiness. I want to make two remarks. 
Firstly, after diagnosing that the patient developed an acute dystonia due to trifluoperazine, the authors write that after withdrawal of trifluoperazine the symptoms subsided over the following week. An anticholinergic drug would most likely have solved the problem and no change of neuroleptic would have been necessary.

The second remark is about the use of the term "Pisa syndrome". The authors write "there are also strong resemblances to the two adult cases reported by Yassa (1985) who also responded to drug withdrawal, having failed to improve with anticholinergic medication". However, Yassa's cases were not acute dystonias but tardive dystonias. Tardive dystonia is quite different from acute dystonia in clinical course, time course in relation to drug exposure, response to anticholinergic treatment, and response to drug withdrawal (Burke et al, 1982; Kang et al, 1988).

Cunningham Owens (1990) declares that the name "Pisa syndrome" is best dropped because it is not a separate entity; we have also left out this term in a review article which is currently under preparation.

Although "pisa syndrome" may be a romantic name it is not well defined and is, therefore, superfluous.

Burke, R. E., FAhn, S., Jankovic, J. H., el al (1982) Tardive dystonia: late-onset and persistent dystonia caused by antipsychotic drugs. Neurology, 32, $1335-1346$.

CUNNINGHam OWENS. D. G. (1990) Dystonia-a potential psychiatric pitfall. British Journal of Psychiatry. 156, 620-634.

KANG, U.J., BURKE, R.E. \& FAHN. S. (1988) Tardive dystonia. Advances in Neurology: 50, 415-429.

YASSA. R. (1985) The pisa syndrome: a report of two cases. British Journal of Psychiatry, 146, 93-95.

Dr David Ricardo Capriles Clinic

P. R. VAN HARTEN

Mohikanenweg 8

Curaçao

Netherlands Antilles

\section{Towards a culture-specific psychotherapy}

SIR: The Western model of psychotherapy, adopted and practised in most countries, does not adequately apply to patients of other cultures. It mostly uses exploratory techniques. Individuals in other cultures are less likely to use the strategies of thinking that would allow effective use of such technique. It is possible that individuals of non-Western cultures use different cognitive processes from those used in Western countries.

In our early practice of psychotherapy at the King Faisal Specialist Hospital and Research Centre in Saudi Arabia, we applied the Western model unchanged. We soon realized that our patients differed from Western patients in several respects. They showed difficulty in handling interpretations that required reflection, and in relating to or examining inner experiences. Traditional methods of putting suggestions as questions were incorrectly understood. 'Why' was always taken in a legalistic sense of right and wrong. Patients were very definite and certain in their ideas, and had difficulty in perceiving problems from a different perspective. They had difficulties in considering alternative viewpoints. There was overlap between their wishes and their account of their experiences. Patients were capable of giving a great deal of sympathy, but showed little empathy. They had difficulty controlling their inner needs and sought immediate satisfaction with little regard to the realities of the situation.

It was necessary to alter our approach, and we increasingly use a behavioural model.

Detailed discussions with many practicing psychotherapists confirmed that they use comparable techniques to our own; however, we were unclear of the exact theoretical model in action.

The cognitive processes used by our patients are similar to those described by Piaget as functioning at pre-formal operational levels. The preponderance of these cognitive processes within our patient population made us wonder if what we were observing was indeed a cultural phenomenon. Initially, we questioned whether such a model of psychological development could be used to describe cultural development. To examine this possibility, we looked into Arabic literature and studies of linguistics, and we re-examined closely the social interaction and moral behaviour of individuals within the culture. The cognitive processes in use within the culture as a whole, and the characteristics of behaviour of individuals were strikingly similar to the pre-formal operational levels in Piaget's stipulations.

Can it be assumed that there are pre-operational cultures, concrete operational cultures and formal operational cultures? We believe it can.

This has been our experience with psychotherapy in our institutions. There is a need for a new theoretical model to guide psychotherapeutic practice in non-Western cultures. This need requires collective transcultural effort. We would, therefore, like to invite interested psychiatrists from Western and nonWestern cultures to share their experiences with us. We will be pleased to receive their comments, either by direct personal contact or through the medium of this journal.

ESMAT El-SHERBinI Kutaiba Chaleby

Department of Medicine

King Faisal Specialist Hospital and Research Centre Riyadh, Saudi Arabia 